\title{
\begin{tabular}{l|l} 
Mitraries & DSpace@MIT
\end{tabular}
}

\author{
MIT Open Access Articles
}

\section{Nanoscale mapping of temperature and defect evolution inside operating AlGaN/GaN high electron mobility transistors}

The MIT Faculty has made this article openly available. Please share how this access benefits you. Your story matters.

Citation: Lin, Chung-Han et al. “Nanoscale mapping of temperature and defect evolution inside operating AlGaN/GaN high electron mobility transistors." Applied Physics Letters 95.3 (2009): 033510-3. (c) 2009 American Institute of Physics

As Published: http://dx.doi.org/10.1063/1.3189102

Publisher: American Institute of Physics

Persistent URL: http://hdl.handle.net/1721.1/59415

Version: Final published version: final published article, as it appeared in a journal, conference proceedings, or other formally published context

Terms of Use: Article is made available in accordance with the publisher's policy and may be subject to US copyright law. Please refer to the publisher's site for terms of use. 


\title{
Nanoscale mapping of temperature and defect evolution inside operating AIGaN/GaN high electron mobility transistors
}

\author{
Chung-Han Lin,, ${ }^{1, a)}$ T. A. Merz, ${ }^{2}$ D. R. Doutt, ${ }^{2}$ M. J. Hetzer, ${ }^{2}$ Jungwoo Joh, $^{3}$ \\ Jesús A. del Alamo, ${ }^{3}$ U. K. Mishra, ${ }^{4}$ and L. J. Brillson ${ }^{1,2,5}$ \\ ${ }^{1}$ Department of Electrical and Computer Engineering, The Ohio State University, Columbus, \\ Ohio 43210, USA \\ ${ }^{2}$ Department of Physics, The Ohio State University, Columbus, Ohio 43210, USA \\ ${ }^{3}$ Microsystems Technology Laboratories, MIT, Cambridge, Massachusetts 02139, USA \\ ${ }^{4}$ Department of Electrical and Computer Engineering and Department of Materials, UC-Santa Barbara, \\ California 93106, USA \\ ${ }^{5}$ Center for Materials Research, The Ohio State University, Columbus, Ohio 43210, USA
}

(Received 22 May 2009; accepted 7 July 2009; published online 22 July 2009)

\begin{abstract}
We used depth-resolved microcathodoluminescence spectroscopy (DRCLS) and Kelvin probe force microscopy (KPFM) to measure and map the temperature distribution and defect generation inside state-of-the-art AlGaN/GaN-based high electron mobility transistors (HEMTs) on a scale of tens of nanometers during device operation. DRCLS measurements of near band edge energies across the HEMT's source-gate-drain regions reveal monotonic temperature increases across the submicron gate-drain channel, peaking under the drain side of the gate. DRCLS defect emissions mapped laterally and localized depthwise near the two-dimensional electron gas interface increase with device operation under the drain-side gate and correlate with higher KPFM surface potential maps. (C) 2009 American Institute of Physics. [DOI: 10.1063/1.3189102]
\end{abstract}

GaN-based high electron mobility transistors (HEMTs) have gained considerable importance as high power, high frequency devices. ${ }^{1-4}$ However, high current density in the channel region leads to significant Joule heating that degrades device performance by decreasing carrier mobility, ${ }^{5-9}$ thermal conductivity, and thermodynamic stability. Nanoscale-resolved measurements of temperature, defects, and electronic properties inside operating HEMT structures can reveal physical mechanisms of degradation under realistic conditions. Here we use a combination of laterally and depth-resolved microcathodoluminescence spectroscopy (DRCLS) (Ref. 10) with atomic force microscopy (AFM) and Kelvin force probe microscopy (KPFM) to monitor temperature, defect energies and densities, and electric potential across source-gate-drain channels of state-of-the-art AlGaN/ GaN HEMTs grown on both sapphire and SiC. Temperature and defect emission maps are localized laterally and depthwise on a scale of tens of nanometers near the twodimensional electron gas (2DEG) interface.

$\mathrm{AlGaN} / \mathrm{GaN}$ device temperatures have been measured previously by visible or ultraviolet micro-Raman methods ${ }^{11-16}$ and scanning thermal microscopy. ${ }^{17}$ Both experimental $^{11,12,14-16}$ and simulation ${ }^{6,12,15,16}$ results show that hot spots and dislocations form near the gate on the drain side. Semiconductor dislocations ${ }^{18}$ and defect states ${ }^{19}$ may degrade mobility, heat distribution, and device characteristics. Accurate spatially localized temperature and materials measurements are important for device modeling and failure analysis. However, submicron lateral and nanoscale layer HEMT features can present challenges in some cases due to diffraction and extended absorption depths, respectively.

\footnotetext{
${ }^{a)}$ Electronic mail: chunghan.lin@gmail.com.
}

We present results for two sets of $\mathrm{AlGaN} / \mathrm{GaN}$ HEMTs, termed U-08 and M-01. U-08 heterostructures grown by metal-organic chemical vapor deposition consist of unintentionally doped (UID) GaN on a sapphire substrate followed by a $0.7 \mathrm{~nm}$ AlN interfacial layer, $40 \mathrm{~nm}$ layer of $\mathrm{Al}_{0.22} \mathrm{Ga}_{0.78} \mathrm{~N}, 10 \mathrm{~nm}$ silicon-doped $\left(7 \times 10^{18} \mathrm{~cm}^{-3}\right)$ graded $(0 \leq x \leq 0.22) \mathrm{Al}_{x} \mathrm{Ga}_{1-x} \mathrm{~N}$, and a $250 \mathrm{~nm}$ UID GaN cap. The UID GaN cap layer is etched away to form source, drain, gate contact, and an "extrinsic drain-source" region, defined as those regions between gate and drain and between gate and source. M-01 consists of nominally the same layer sequence but grown on $\mathrm{SiC}$ with a $640 \mathrm{~nm} \mathrm{Si}_{3} \mathrm{~N}_{4}$ passivation layer versus the UID GaN cap.

A JEOL JAMP-7800F ultrahigh vacuum (UHV) scanning electron microscope (SEM) with nm-scale resolution excited electron-hole pair recombination and cathodoluminescence $(\mathrm{CL})$ with electron beam energies $E_{B}$ ranging from 5 to $20 \mathrm{keV}$. We used layer-dependent Monte Carlo simulations ${ }^{20}$ to select $E_{B}$ that probe near the $\mathrm{AlGaN} / \mathrm{GaN}$ 2DEG heterointerface. An Oxford MonoCL grating monochromator and Hamamatsu photomultiplier tube collected optical emission signals from samples at low $(\sim 12 \mathrm{~K})$ and room temperature. A Park XE-70 AFM/KPFM provided simultaneous topography and potential maps. ${ }^{21}$ An Agilent 4145B analyzer enabled electrical stress measurements.

Figure 1 shows the U-08 temperature distribution between gate and drain, termed the "extrinsic drain region," with beam current $I_{B} \sim 0.5 \mathrm{nA}$. Peak electron-hole pair excitation depth $U_{0}$ derived from Monte Carlo simulation is $\sim 125 \mathrm{~nm}$ for $E_{B}=5 \mathrm{keV}$, just $75 \mathrm{~nm}$ below the 2DEG channel region. DRCLS provides local temperatures since the $\mathrm{GaN}$ near band edge (NBE) emission energy varies as $E_{g}(T)=E_{g}(0)-\left(5.98+0.12 \times 10^{-4} \mathrm{eV} / \mathrm{C}\right) T$ for temperature $T$ and band gap $E_{g} .{ }^{22}$ Under low power (source-drain voltage $V_{\mathrm{DS}}=3.5 \mathrm{~V}$, gate-source voltage $V_{\mathrm{GS}}=-2 \mathrm{~V}$, and drain- 


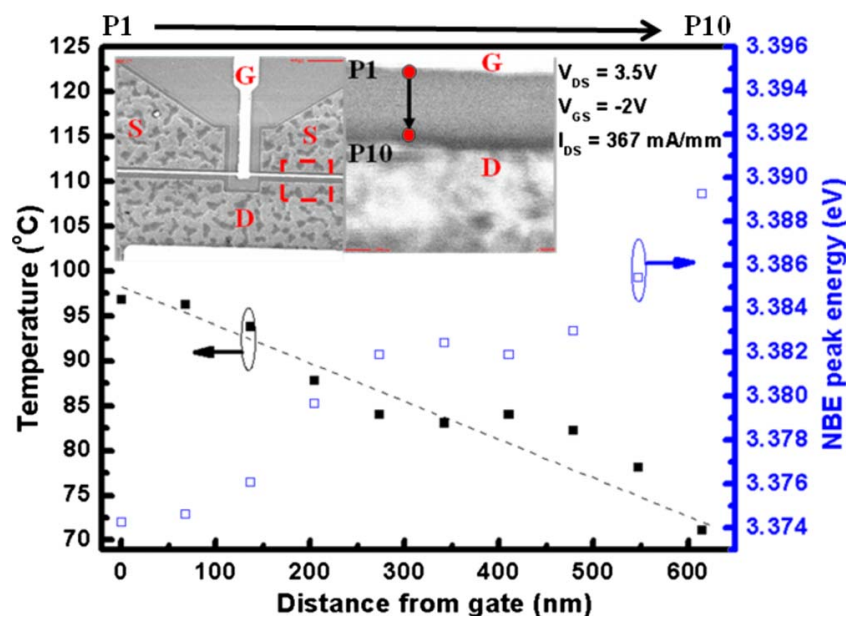

FIG. 1. (Color online) Temperature distribution of U-08 operated at $V_{\mathrm{DS}}$ $=3.5 \mathrm{~V}$ and $V_{\mathrm{GS}}=-2 \mathrm{~V}$. P1 is nearest the gate while P10 is furthest away. Inset photograph shows SEM image of source-drain region measured.

source current $I_{\mathrm{DS}}=367 \mathrm{~mA} / \mathrm{mm}$ ), Fig. 1 shows temperature increasing from drain to gate along ten equally spaced points along a perpendicular to and between gate and drain, consistent with micro-Raman results ${ }^{11-14}$ and other techniques but with dramatically higher spatial and depth resolution. Localized electron beam heating produces no measurable NBE temperature rise at room temperature. At $I_{B}=0.5 \mathrm{nA}$ and $E_{B}=5 \mathrm{keV}$, induced beam currents are $\sim 0.2 \mu \mathrm{A}$, negligible compared to our device currents. Piezoelectric strain can shift NBE energies by $24 \mathrm{meV} / \mathrm{GPa}$ (Ref. 23$)=40^{\circ} \mathrm{C} / \mathrm{GPa}$, but $V_{\mathrm{SD}}=40 \mathrm{~V}$ produces strains of $\sim 0.4 \mathrm{GPa}$ at the drain ${ }^{24}$ that extrapolate linearly to $<1{ }^{\circ} \mathrm{C}$ for $V_{\mathrm{SD}}=6 \mathrm{~V}$ here.

Figure 2(a) shows the SEM image of another U-08 GaN HEMT operated to failure $\left(V_{\mathrm{DS}}=3.5 \mathrm{~V}, V_{\mathrm{GS}}=0 \mathrm{~V}, I_{\mathrm{DS}}\right.$ $=633 \mathrm{~mA} / \mathrm{mm}, 4 \mathrm{~h}$ ) in UHV. AFM revealed a $100-150 \mathrm{~nm}$ deep, $\sim 60 \mathrm{~nm}$ wide crater in the extrinsic drain region. A $\mathrm{CL}$ map of NBE $(\sim 3.42 \mathrm{eV}$ at $12 \mathrm{~K})$ emission in Fig. 2(b)

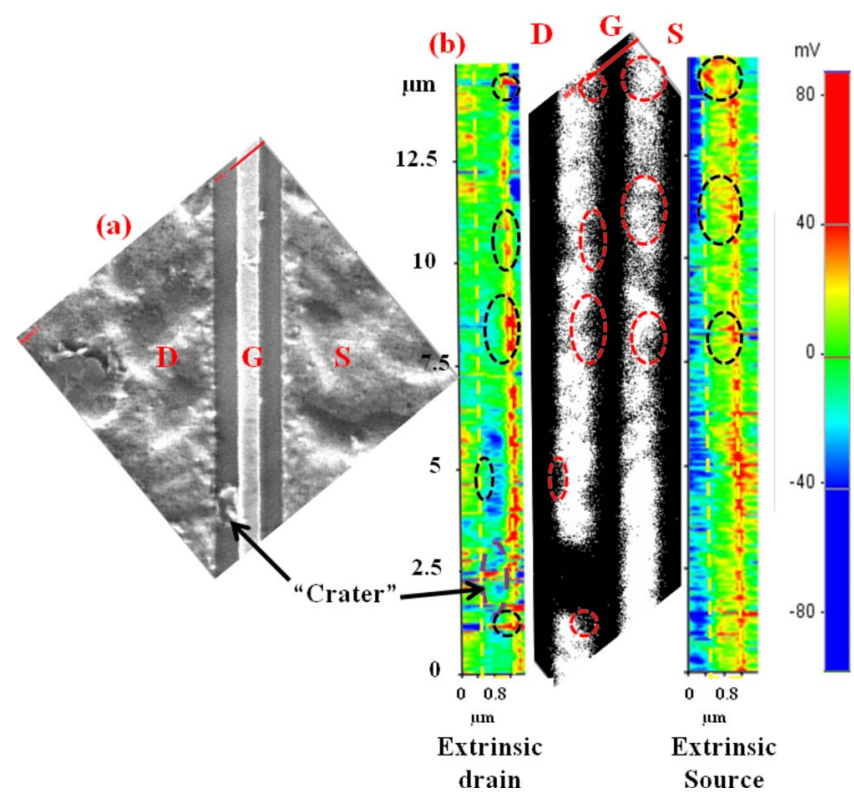

FIG. 2. (Color online) For U-08, (a) SEM image shows the Source (s), Gate (g), and Drain (d) region. (b) CL map of NBE emission (middle) and KPFM result (side) taken in the same (a) region. Red dashed circles show some of the same higher defect, higher potential region for CL and KPFM map, respectively, where devices degrade faster.
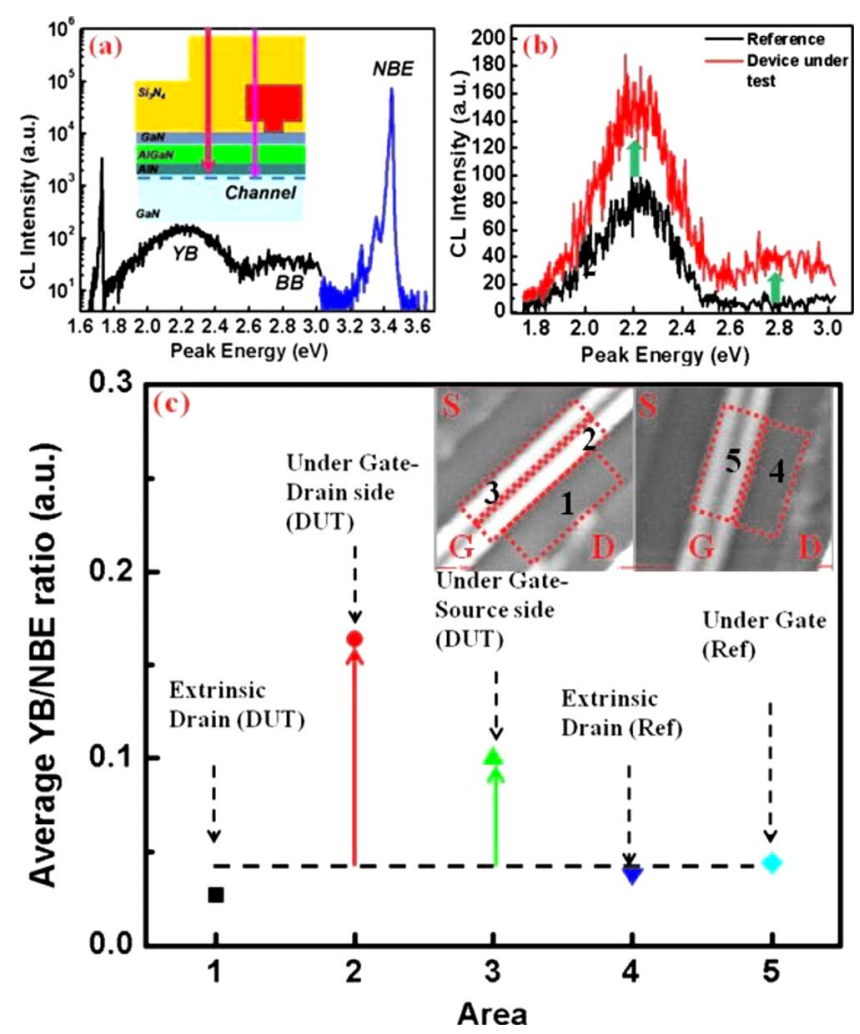

FIG. 3. (Color online) DRCLS results for M-01 sample, (a) DRCLS spectrum from under the gate overhang before operation; Inset: schematic of $\mathrm{M}-01$ cross section. Arrow indicates incident electron beam path through gate overhang at $E_{B}=20 \mathrm{keV}$. (b) increase in $2.2 \mathrm{eV} \mathrm{YB} \mathrm{emissions} \mathrm{before}$ [also (a)] and after $12 \mathrm{~h}, V_{\mathrm{D}}=10 \mathrm{~V}, V_{\mathrm{G}}=-2 \mathrm{~V}, 476 \mathrm{~mA} / \mathrm{mm}$ operation. (c) Position-dependent average $\mathrm{YB} / \mathrm{NBE}$ ratios showing largest increase under drain-side gate after $12 \mathrm{~h}$ operation. Inset shows SEM image and definition of areas $1-5$.

shows (i) NBE disappearance at the failure region and (ii) variations in NBE intensity within the extrinsic drain and source. Bright CL map areas exhibit 1000-fold higher NBE intensity than dark areas in the extrinsic drain versus uniform intensity of the virgin sample. NBE intensity decreases apparent in Fig. 2(b) signify free carrier recombination at subband gap energies.

KPFM maps in Fig. 2(b) show nonuniform potential distribution. Dashed rectangles define the same extrinsic drain and source regions as in Fig. 2(a), while an ellipse outlines the "crater." Surface potentials vary by more than $100 \mathrm{meV}$ within the unbiased extrinsic drain and source regions, with higher potentials in general corresponding to regions of reduced NBE emission (higher defect density). Surface photovoltage spectra (SPS) indicate defect levels $2.2 \mathrm{eV}$ below the conduction band increasing in these regions. ${ }^{25}$

Varying $E_{B}$ allowed us to preferentially excite not only unpassivated extrinsic drain and source regions but also regions passivated with dielectrics or shadowed by gate overhangs. Figure 3(a) shows a DRCL spectrum with features at $h \nu=3.4 \mathrm{eV}$ (NBE), $2.2 \mathrm{eV}$ "yellow band" (YB), and 2.8-3.2 $\mathrm{eV}$ "blue band" (BB) (Ref. 26) taken from the drain-side gate overhang region for the virgin M-01 HEMT. YB emission is typically associated with $\mathrm{Ga}$ vacancies or $\mathrm{Ga}$ vacancyimpurity complexes in $\mathrm{GaN}^{23}$ The Fig. 3(a) inset illustrates the cross sectional HEMT passivation layer, gate and active layer geometry through which the electron beam can penetrate. For M-01, $E_{B}=8$ and $20 \mathrm{keV}$ produce electron-hole 
pairs peaking at $\sim 660$ and $1300 \mathrm{~nm}$ at the 2DEG layer next to versus through the gate overhang, respectively. Figure 3(b) illustrates the increase in YB defect emission after $12 \mathrm{~h}$ device operation (termed "Device under test") at $V_{\mathrm{DS}}=10 \mathrm{~V}$, $V_{\mathrm{GS}}=-2 \mathrm{~V}$, and $I_{\mathrm{DS}}=476 \mathrm{~mA} / \mathrm{mm}$ (red curve) compared with emission (black) at the same location before operation ("Reference device").

Figure 3(c) displays the changes in YB to NBE defect emissions ratio versus position within the source-gate-drain region. Each data point representing the ratio $I(\mathrm{YB}) / I(\mathrm{NBE})$ of defect versus NBE emission intensities corresponds to an average of spectra at the same distance from and along the width of the gate. Figure 3(c) shows that YB under the gate on the drain side increases by a factor of 3.5 compared with $\sim 2.2 x$ under the gate on the source side and very little within the extrinsic drain region. In contrast, averaged $\mathrm{BB} / \mathrm{NBE}$ ratios shows no strong correlation with position. This result shows that device operation can increase defect emissions within $\mathrm{AlGaN} / \mathrm{GaN}$ HEMTs. Figure 3(c) shows that the growth of such defect concentrations varies systematically across the source-drain-channel region. Furthermore, device operation affects defect changes preferentially. YB defect emission exhibits much larger and systematic increases than defects characterized by BB emission, indicating a link between this defect and device degradation. This $2.2 \mathrm{eV}$ YB defect emission is attributed to transitions involving deep levels $2.2 \mathrm{eV}$ below the conduction band, ${ }^{26}$ consistent with SPS measurements for U-08. ${ }^{25}$ Such defect formation during device operation could alter Fermi level positions, band bending, and hence potential within the surface layer. Consistent with a wealth of electrical stress studies, ${ }^{27}$ these electrical and material changes may contribute to degradation of HEMT performance and ultimately device failure. After stressing, saturation current decreased $4 \%-6 \%$ and gate leakage current increased one order of magnitude, consistent with previous literature. Further details will be published elsewhere.

In summary, we have demonstrated the utility of electron microscope-based CL spectroscopy to probe temperature distributions on a nanometer scale inside state-of-the-art HEMTs during device operation. Consistent with previously published data, temperatures increase from drain to gate, reaching maximum values under the drain-side gate. Depthresolved defect emission is distributed nonuniformly across the source-gate-drain region. Specific defect emissions related to Ga vacancies are found to increase with device operation, increasing from drain to gate, indicating that longer operation promotes increased YB defect formation. Overall, the nanoscale, depth-resolved optical, and scanned probe techniques reported here can be used to reveal and in principle predict physical mechanisms of AlGaN/GaN HEMT degradation under realistic conditions.
This work is supported by the Office of Naval Research Grant No. N00014-08-1-0655 (Paul Maki and Harry Dietrich).

${ }^{1}$ Y. F. Wu, D. Kapolnek, J. P. Ibbetson, P. Parikh, B. P. Keller, and U. K. Mishra, IEEE Trans. Electron Devices 48, 586 (2001).

${ }^{2}$ U. K. Mishra, P. Parikh, and Y.-F. Wu, Proc. IEEE 90, 1022 (2002).

${ }^{3}$ O. Ambacher, B. Foutz, J. Smart, J. R. Shearly, N. G. Weimann, K. Chu, M. Murphy, A. J. Sierakowski, W. J. Schaff, L. F. Eastman, R. Dimitrov, A. Mitchell, and M. Stutzmann, J. Appl. Phys. 87, 334 (2000).

${ }^{4}$ O. Ambacher, J. Smart, J. R. Shealy, N. G. Weimann, K. Chu, M. Murphy, W. J. Schaff, L. F. Eastman, R. Dimitrov, L. Wittmer, M. Stutzmann, W. Rieger, and J. Hilsenbeck, J. Appl. Phys. 85, 3222 (1999).

${ }^{5}$ Y. Chang, Y. Zhang, Y. Zhang, and K. Y. Tong, J. Appl. Phys. 99, 044501 (2006).

${ }^{6}$ W. D. Hu, X. S. Chen, Z. J. Quan, C. S. Xia, W. Lu, and P. D. Ye, J. Appl. Phys. 100, 074501 (2006).

${ }^{7}$ S. A. Vitusevich, S. V. Danylyuk, N. Klein, M. V. Petrychuk, A. Yu. Avksentyev, V. N. Sokolov, V. A. Kochelap, A. E. Belyaev, V. Tilak, J. Smart, A. Vertiatchikh, and L. F. Eastman, Appl. Phys. Lett. 82, 748 (2003).

${ }^{8}$ N. Braga, R. Mickevicius, R. Gaska, X. Hu, M. S. Shur, M. Asif Khan, G. Simin, and J. Yang, J. Appl. Phys. 95, 6409 (2004).

${ }^{9}$ N. Braga, R. Mickevicius, R. Gaska, M. S. Shur, M. Asif Khan, and G. Simin, Appl. Phys. Lett. 85, 4780 (2004).

${ }^{10}$ L. J. Brillson, J. Vac. Sci. Technol. B 19, 1762 (2001).

${ }^{11}$ M. Kuball, J. M. Hayes, M. J. Uren, T. Martin, J. C. H. Birbeck, R. S Balmer, and B. T. Hughes, IEEE Electron Device Lett. 23, 7 (2002).

${ }^{12}$ M. Kuball, S. Rajasingam, A. Sarua, M. J. Uren, T. Martin, B. T. Hughes, K. P. Hilton, and R. S. Balmer, Appl. Phys. Lett. 82, 124 (2003).

${ }^{13}$ J. W. Pomeroy, M. Kuball, D. J. Wallis, A. M. Keir, K. P. Hilton, R. S. Balmer, M. J. Uren, T. Martin, and P. J. Heard, Appl. Phys. Lett. 87, 103508 (2005).

${ }^{14}$ J. W. Pomeroy, M. Kuball, M. J. Uren, K. P. Hilton, R. S. Balmer, and T. Martin, Appl. Phys. Lett. 88, 023507 (2006).

${ }^{15}$ I. Ahmad, V. Kasisomayajula, M. Holtz, J. M. Berg, S. R. Kurtz, C. P. Tigges, A. A. Allerman, and A. G. Baca, Appl. Phys. Lett. 86, 173503 (2005).

${ }^{16}$ I. Ahmad, V. Kasisomayajula, D. Y. Song, L. Tian, J. M. Berg, and M. Holtz, J. Appl. Phys. 100, 113718 (2006).

${ }^{17}$ R. Aubry, J.-C. Jacquet, J. Weaver, O. Durand, P. Dobson, G. Mills, M.-A. di Forte-Poisson, S. Cassette, and S.-L. Delage, IEEE Trans. Electron Devices 54, 385 (2007).

${ }^{18}$ J. Zou, D. Kotchetkov, A. A. Balandin, D. I. Florescu, and F. H. Pollak, J. Appl. Phys. 92, 2534 (2002).

${ }^{19}$ W. Saito, M. Kuraguchi, Y. Takada, K. Tsuda, I. Omura, and T. Ogura, IEEE Trans. Electron Devices 52, 159 (2005).

${ }^{20}$ http://www.gel.usherbrooke.ca/casino.

${ }^{21}$ D. R. Doutt, C. Zgrabik, H. L. Mosbacker, and L. J. Brillson, J. Vac. Sci. Technol. B 26, 1477 (2008).

${ }^{22}$ A. P. Young and L. J. Brillson, Appl. Phys. Lett. 77, 699 (2000).

${ }^{23}$ W. Rieger, T. Metzger, H. Angerer, R. Dimitrov, O. Ambacher, and M. Stutzmann, Appl. Phys. Lett. 68, 970 (1996).

${ }^{24}$ A. Sarua, H. Ji, M. Kuball, M. J. Uren, T. Martin, K. J. Nash, K. P. Hilton, and R. S. Balmer, Appl. Phys. Lett. 88, 103502 (2006); A. Sarua, T. Batten, H. Ji, M. J. Uren, T. Martin, and M. Kuball, CS Mantech Conference, May 2009 (unpublished).

${ }^{25}$ C.-H. Lin, T. A. Merz, D. R. Doutt, J. A. del Alamo, U. K. Mishra, and L. J. Brillson (unpublished).

${ }^{26}$ S. T. Bradley, A. P. Young, L. J. Brillson, M. J. Murphy, W. J. Schaff, and L. F. Eastman, IEEE Trans. Electron Devices 48, 412 (2001).

${ }^{27}$ J. Joh and J. A. del Alamo, IEEE Electron Device Lett. 29, 287 (2008), and references therein. 